\title{
EL PAISAJE VEGETAL DE LA DEPRESIÓN DE VERA DURANTE LA PREHISTORIA RECIENTE. UNA APROXIMACIÓN DESDE LA ANTRACOLOGÍA
}

\author{
THE VEGETATION OF THE VERA BASIN IN RECENT PREHISTORY. \\ AN ANTHRACOLOGICAL APPROACH
}

M. ${ }^{a}$ OLIVA RODRÍGUEZ ARIZA (*)

\begin{abstract}
RESUMEN
A partir de la Antracología se estudian y evalúan los resultados obtenidos en tres yacimientos de la Edad del Cobre y dos de la Edad del Bronce situados en la Depresión de Vera, una de las comarcas más áridas del Sureste de la Península Ibérica. A partir de los resultados obtenidos podemos decir que la vegetación durante el III milenio a.n.e. estaba dominada por comunidades arbustivas instaladas en el centro o zonas bajas de la Depresión de Vera, mientras que las formaciones arbóreas se situarían en los piedemontes de las sierras cercanas. Durante el II milenio parece que existe un progresivo aumento de la aridez, que hace que las formaciones arbustivas vayan siendo sustituidas por matorrales abiertos y las formaciones arbóreas se queden relegadas en las sierras.
\end{abstract}

\begin{abstract}
Anthracological techniques have been used to study and evaluate the remains at three sites from the CopperAge and two from the Bronze Age, all situated in the Vera Basin, one of the most arid areas in the southeast of the Iberian Peninsula. The results indicate that the vegetation during the third millennium B.C. was dominated by shrub communities in the central and low zones of this basin, while tree formations predominated in the foothills of the nearby mountains. During the second millennium B.C., aridity appears to have increased progressively, causing the shrubby communities to be replaced by open scrublands and relegating the tree formations to the mountain slopes.
\end{abstract}

(*) Centro Andaluz de Arqueología Ibérica. Universidad de Jaén. Edif. B-1. 23071 Jaén. Tel. 953 212132. Correo electrónico: moliva@ujaen.es.

El artículo fue remitido en su versión final el 22-III-00.
Palabras clave: Antracología. Edad del Cobre. Edad del Bronce. Sureste de la Península Ibérica.

Key words: Anthracology. CopperAge. Bronze Age. Southeastern Iberian Peninsula.

\section{INTRODUCCIÓN}

LaAntracología es una joven ciencia y su papel en la reconstrucción de la vegetación y el clima ha sido subestimado durante mucho tiempo, indicando siempre las limitaciones y no las ventajas (Fernández, 1989). El esquema general de la historia de la vegetación cuaternaria ha sido siempre realizada por la Palinología. Últimamente los aportes de la Antracología para el conocimiento de la vegetación han comenzado a ser valorados y es evidente que la Palinología y laAntracología son métodos complementarios (Leroi-Gourhan, 1992), que combinados proveen de una reconstrucción real y detallada de la vegetación del pasado.

La profusión de estudios antracológicos en el sur de Francia ha facilitado el establecimiento de una zonación biocronológica de los últimos doce milenios (Vernet et alii, 1987; Vernet y Thiébault, 1987). Esta zonación, al definir las cuatro fases principales, tiene en cuenta tanto los factores climáticos como las relaciones hombre-vegetación. La realización de análisis antracológicos dentro del ámbito del Mediterráneo Occidental es cada vez más frecuente, por lo que los resultados obtenidos se pueden correlacionar con la zonación biocronológica del sur de Francia, aunque las diferencias latitudinales y altitudinales habrán de ser tomadas en cuenta en cada región o comarca analizada. 


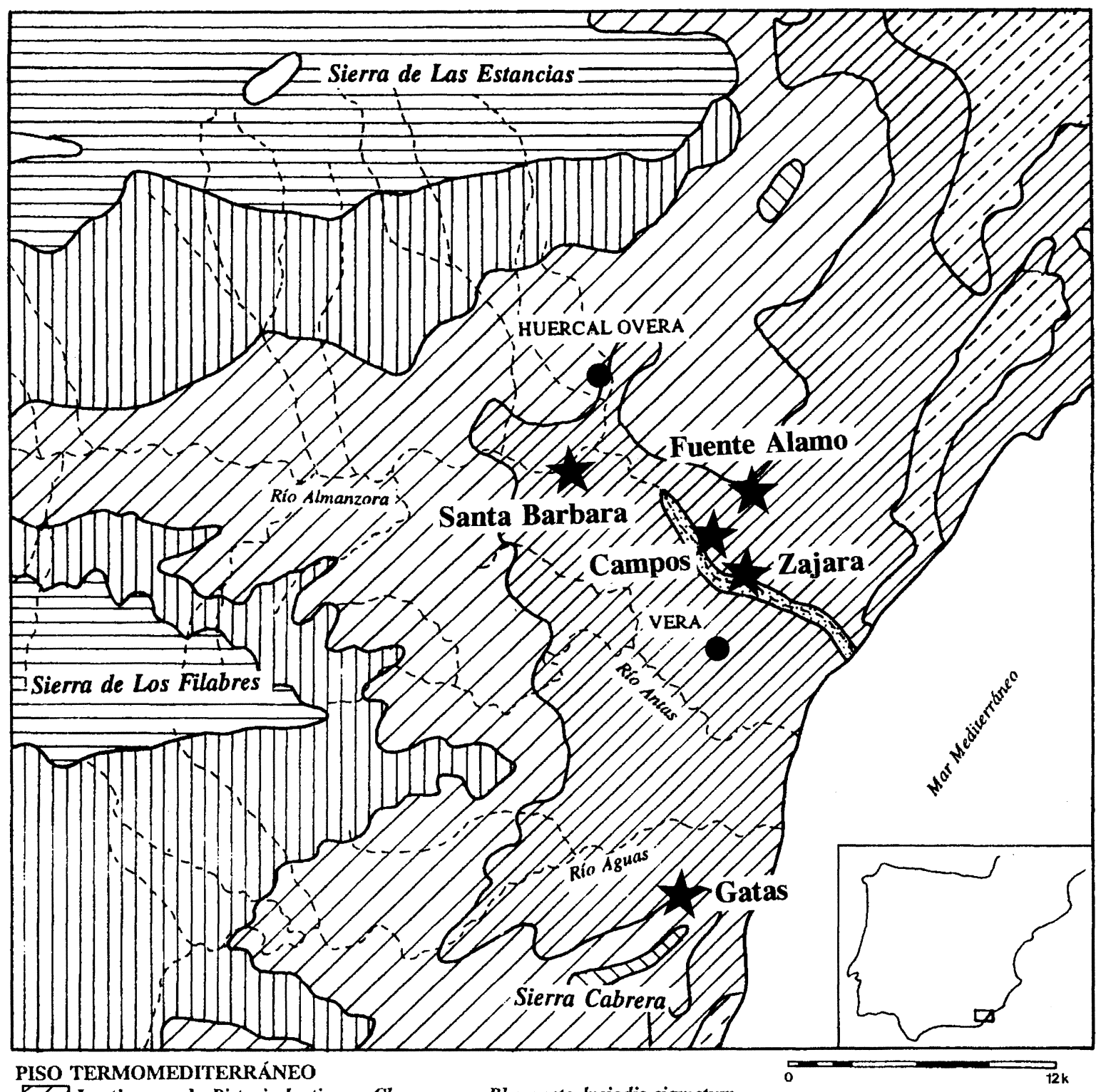

7 Lentiscares de Pistacia lentiscus. Chamaeropo-Rhamneto lyciodis sigmetum.

D. Cornicales de Periploca angustifolia.Mayteno europaei-Periploceto angustifoliae s.

Z7 Espinal de azufaifos. Zizipheto loti sigmetum.

$\Delta 17$ Encinares basófilos termófilos.Smilaci mauritanicae-Querceto rotundifoliaes.

PISO MESOMEDITERRÁNEO

[II] Coscojares. Rhamno lycioidi-Querceto cocciferae sigmetum.

PISO SUPRAMEDITERRÁNEO

$\square$ Encinares silicícolas. Adenocarpo decorticanti-Querceto rotundifoliae sigmetum.

Encinares silicículas mesófilos con Retama sphaerocarpa

Geoseries edafófilas mediterráneas

Geomegaseries riparias mediterráneas y regadíos.

Fig. 1. Mapa de series de vegetación (Rivas Martínez, 1988) y ubicación de los yacimientos ( $\star$ ).

T. P., 57, n. ${ }^{\circ} 1,2000$ 
Hablar del medioambiente del Sureste de la Península Ibérica es hablar de un mosaico de ambientes diferentes, donde se pueden encontrar varios ombroclimas, desde las zonas semiáridas de Cabo de Gata y las depresiones intrabéticas de Baza y Guadix, hasta las zonas húmedas de las Sierras de Segura y Cazorla, cinco de los seis pisos bioclimáticos determinados para la región mediterránea (desde el Termomediterráneo de las zonas litorales al oromediterráneo de las cumbres de Sierra Nevada) y multitud de asociaciones vegetales que los botánicos han agrupado en tres provincias corológicas (en función de la vegetación): Bética, Murciano-almeriense y Castellano-maestrazgo-manchega. En este artículo nos centramos en la denominada Depresión deVera al Norte de la provincia deAlmería (Fig. 1), una de las comarcas más áridas del Sureste peninsular. Por ello, hemos creído importante hacer una breve descripción de sus características bioclimáticas actuales para una mejor comprensión y contrastación de los resultados obtenidos en los análisis antracológicos.

\section{YACIMIENTOS Y BIOGEOGRAFÍA}

Los yacimientos con análisis antracológicos en la Depresión de Vera han sido excavados en las dos últimas décadas, por varios equipos de investigación. Los carbones de los yacimientos de Campos, Zajara y Santa Bárbara, situados en la Cuenca del Bajo Almanzora y excavados por un equipo de la Universidad de la Laguna, fueron estudiados por nosotros (1). El antracoanálisis de Fuente Álamo fue realizado por Schoch y Schweingruber (1982) y el de Gatas por Rowena Gale (1999) (2).

Biogeográficamente la zona del BajoAlmanzora pertenece a la región Mediterránea y dentro de la tipología biogeográfica o corológica a la provincia Murciano-Almeriense en su sector Almeriense (Rivas Martínez, 1988; Peinado Lorca y Rivas Martínez, 1987).

Bioclimáticamente están representados en esta

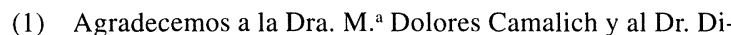
mas Martín el haber puesto a nuestra disposición el material antracológico de sus excavaciones. El antracoanálisis fue efectuado en el Dpto. de Prehistoria y Arqueología de la Universidad de Granada, a cuyo director, el Dr. F. Molina, queremos expresar nuestra gratitud por su continuo apoyo a nuestra investigación.

(2) Los resultados del antracoanálisis de Gatas, efectuado por R. Gale, nos fueron facilitados por el Dr. V. Lull dentro de nuestra participación en el Proyecto Aguas (Castro et alii, 1998), al cual queremos agradecer las facilidades prestadas para la realización de este artículo. zona los pisos termo-, meso- y supramediterráneos, que en líneas generales vienen a coincidir con los pisos de vegetación de igual nombre (Fig. 1). La mayor o menor continentalidad, que se puede expresar por un mayor o menor frío invernal o calor estival, parece ser el factor que rige en primer término la distribución de las series de vegetación termomediterráneas murciano-almerienses, presentes en el territorio estudiado.

Pasamos a describir brevemente las características de las series de vegetación entorno a los yacimientos estudiados:

- Serie termomediterránea murciano-almeriense semiárida-árida del azufaifo (Ziziphus lotus): Zizipheto loti sigmetum, comunidad de aspecto intrincado y cerrado que favorece la creación de condiciones microclimáticas en su interior, lo que facilita el desarrollo de aquellas especies vegetales más exigentes. Su composición florística en esta zona presenta escasa variabilidad, reduciéndose al siguiente grupo de especies: Ziziphus lotus, Lycium intricatum, Salsola verticilata, Launaea arborecens, Asparagus stipularis y Ballota hirsuta.

El interés tanto de esta comunidad como de sus etapas seriales se sitúa a dos niveles. Por una parte, posee un marcado interés biogeográfico al aportar datos válidos para confirmar la antigua conexión con el Norte de África del Sudeste árido peninsular, y por otra, resulta un bioindicador, valiosísimo, ya que resulta patente que el progresivo avance de estas comunidades o de algunas de sus especies directrices camina paralelo al avance de los procesos de desertización.

- Serie termomediterránea murciano-almeriense semiárida del lentisco (Pistacia lentiscus): Chamaeropo humilis-Rhamneto lycioidis sigmetum. $\mathrm{La}$ vegetación madura es un matorral esclerófilo con lentiscos (Pistacia lentiscus), palmitos (Chamaerops humilis), acebuches (Olea europaea var. sylvestris), bayón, espinos, belcho, algarrobos, esparraguera, etc. Generalmente esta asociación se halla muy alterada, quedando reducida a manchas dispersas de palmitos y espinos negros.

Al Norte de la zona estudiada está presente la serie del cornical, donde no llega a producirse heladas, mientras que en las del lentisco y el azufaifo sí se producen. Las series del cornical y del azufaifo son series más o menos litorales que no exceden el territorio termomediterráneo del sector Almeriense de la provincia murciano-almeriense, en tanto que el lentiscar tiene una distribución más interior. 
Dentro del Piso Mesomediterráneo la serie de vegetación dominante en la zona es la semiárida de la coscoja (Quercus coccifera): Rhamno lycioidiQuerceto cocciferae sigmetum, asociación dominante en las vertientes de la Sierra de Los Filabres y de la Sierra de Las Estancias y, por tanto, un poco alejada de los yacimientos estudiados.

Esta serie corresponde en su etapa madura a bosquetes densos de Quercus coccifera en los que prosperan diversos espinos, sabinas, pinos y otros arbustos mediterráneos (Rhamnus lycioides, Pinus halepensis, Juniperus phoenicea, Juniperus oxycedrus, Daphne gnidium, Ephedra nebrodensis, etc.) y que en áreas particularmente cálidas o en el horizonte inferior mesomediterráneo pueden llevar otros arbustos más termófilos (Pistacia lentiscus, Ephedra fragilis, Asparagus stipularis, etc.).

El rasgo esencial de esta serie es la escasez de las precipitaciones a lo largo del año, en general de tipo semiárido, lo que resulta ser un factor limitante insuperable para que en los suelos no compensados hídricamente puedan prosperar las carrascas ( Quercus rotundifoliae), y, en consecuencia, el óptimo de la serie de vegetación no pueda alcanzar la estructura del bosque planifolio-esclerófilo, sino más bien la de la garriga densa o silvo-estepa (Rivas Martínez, 1988: 118).

La vegetación edafófila o riparia está representada en esta área por la Geoserie de ramblas murciano-almeriense (Glycirrhizo glabrae-Tamaricetum canariensis), asociación instalada sobre suelos algo limosos e integrada por comunidades donde dominan los tarayes (Tamarix canariensis).

Todas estas Series tienen en la actualidad un alto grado de degradación, estando actualmente presentes en la mayor parte del territorio las fases últimas como son los espartales, albardinares, pastizales o tomillares que apenas recubren la superficie. También, gran parte del área está ocupada en la actualidad por cultivos o es objeto de un pastoreo extensivo, que unido a la actividad minera y la fuerte urbanización, sobre todo en la franja litoral, han hecho casi desaparecer la vegetación natural de la zona.

\section{LOS ANÁLISIS ANTRACOLÓGICOS}

Presentamos aquí una valoración de cada uno de los estudios efectuados hasta el momento y realizamos una aproximación al paisaje vegetal de toda la Comarca de Vera durante el III y II milenio a.n.e.

\section{III.1. Interpretación de resultados de yacimientos de la Edad del Cobre}

Los análisis antracológicos de este periodo se han centrado en los yacimientos de Zajara, Campos y Santa Bárbara. En la tabla 1 se muestran los resultados obtenidos en cada uno de ellos.

El número de taxones determinados se eleva a 21 , y si exceptuamos los tres que pueden comprender bajo su denominación a alguno de los otros de la lista (Pinus sp., Pistacia sp. y Rhamnus-Phillyrea), este es de 18. Por tanto, el catálogo florístico proporcionado por el análisis antracológico de los tres yacimientos estudiados está compuesto por dos especies de pinos: el carrasco y el marítimo; por arbustos de gran porte como son el acebuche, la coscoja, el lentisco y la cornicabra; y por especies del matorral fruticoso: salados, jaras, belchos, torviscos, leguminosas, aladiernos y romeros. Junto a esta vegetación climatófila se desarrollan especies que crecen junto a los cursos de agua como son:

\begin{tabular}{|l|c|c|c|c|c|c|}
\hline YACIMIENTOS & \multicolumn{2}{|c|}{ ZAJARA } & \multicolumn{2}{c|}{ CAMPOS } & \multicolumn{2}{c|}{ S. BARBARA } \\
\hline Taxones & $\mathrm{N}^{\circ}$ & $\%$ & $\mathrm{~N}^{\circ}$ & $\%$ & $\mathrm{~N}^{\circ}$ & $\%$ \\
\hline Pinus halepensis & 19 & 4.43 & 29 & 3.24 & 5 & 4.80 \\
\hline Pinus pinaster & - & - & - & - & 1 & 0.97 \\
\hline Pinus sp. & - & - & 1 & 0.12 & 1 & 0.97 \\
\hline Atriplex halimus & 1 & 0.23 & 8 & 0.9 & - & - \\
\hline Cistus sp. & 1 & 0.23 & 6 & 0.67 & - & - \\
\hline Daphne gnidium & - & - & 2 & 0.24 & - & - \\
\hline Ephedra sp. & - & - & 4 & 0.45 & - & - \\
\hline Leguminosae & 2 & 0.46 & 25 & 2.79 & - & - \\
\hline Olea europaea & 239 & 55.70 & 551 & 61.7 & 37 & 35.57 \\
\hline Phillyrea sp. & 1 & 0.23 & 13 & 1.46 & 2 & 1.92 \\
\hline Pistacia lentiscus & 19 & 4.43 & 54 & 6.04 & 10 & 9.61 \\
\hline Pistacia terebinthus & 35 & 8.12 & 48 & 5.37 & 1 & 0.97 \\
\hline Pistacia sp. & 27 & 6.20 & 34 & 3.8 & 10 & 9.61 \\
\hline Quercus ilex-coccifera & 3 & 0.70 & 4 & 0.44 & 9 & 8.65 \\
\hline Rhamnus sp. & - & - & - & - & - & - \\
\hline Rhamnus-Phillyrea & - & - & 1 & 0.12 & - & - \\
\hline Rosmarinus officinalis & 8 & 1.86 & 24 & 2.68 & 5 & 4.80 \\
\hline Fraxinus sp. & 3 & 0.70 & - & - & - & - \\
\hline Populus sp. & 3 & 0.70 & 3 & 0.34 & 1 & 0.97 \\
\hline Salix sp. & 2 & 0.46 & 1 & 0.12 & 8 & 7.69 \\
\hline Tamarix sp. & 34 & 7.91 & 19 & 2.12 & 1 & 0.97 \\
\hline Monocotiledoneae & - & - & 6 & 0.67 & - & - \\
\hline Indeterminadas & 1 & 0.23 & 2 & 0.24 & - & - \\
\hline Indeterminables & 32 & 7.41 & 58 & 6.49 & 13 & 12.5 \\
\hline TOTAL CARBONES & 430 & 100 & 893 & 100 & 104 & 100 \\
\hline No TAXONES & 15 & & 19 & & 13 & \\
\hline
\end{tabular}

Tab. 1. Frecuencias absolutas y relativas de los taxones en los yacimientos en la Edad del Cobre del BajoAlmanzora.

T. P., 57, n. $^{\circ} 1,2000$ 


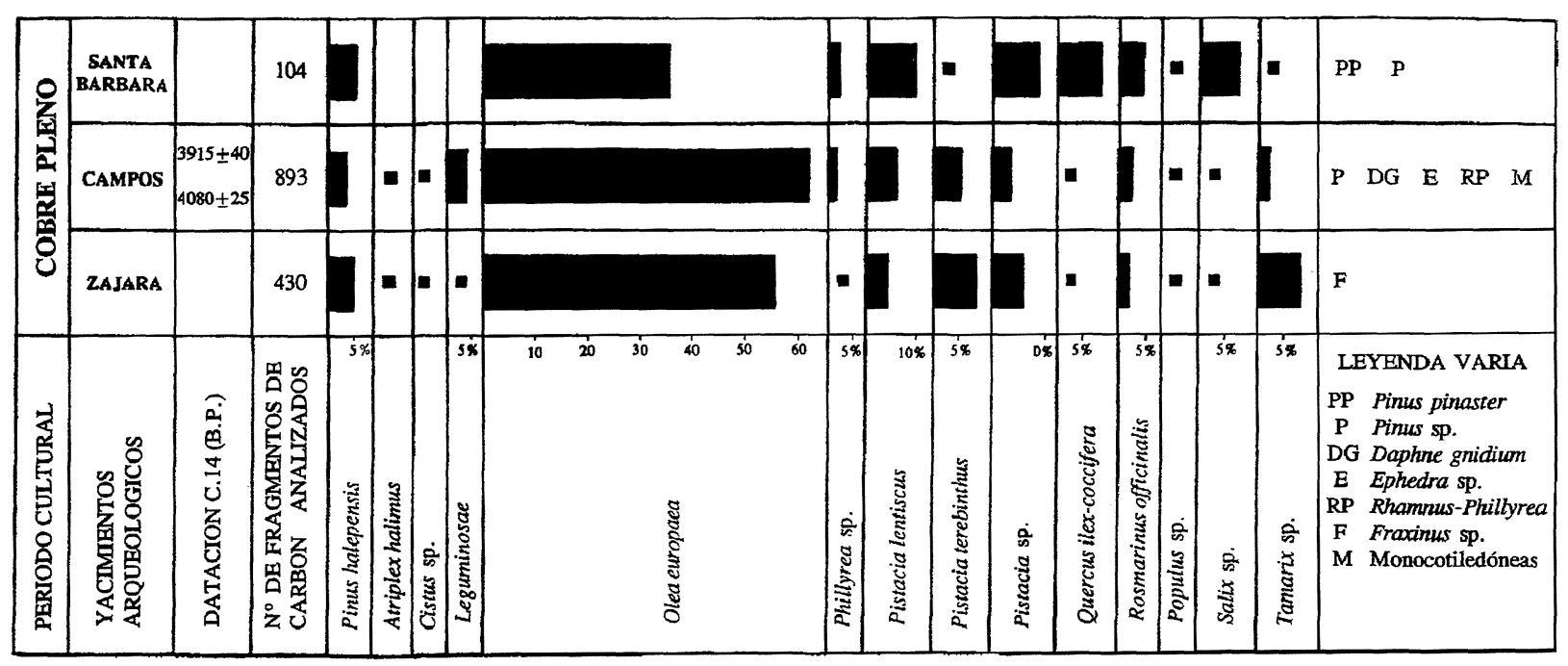

Fig. 2. Histograma antracológico del Bajo Almanzora durante la Edad del Cobre.

fresnos, álamos, sauces y tarayes. También aparecen monocotiledóneas sin determinar, aunque podrían ser restos de cereales y cañas.

A nivel cuantitativo estos taxones muestran una representación muy homogénea entre los tres yacimientos, salvo pequeñas variaciones que más adelante comentaremos (Fig. 2). La especie más representada es el acebuche (Olea europaea var. sylvestris) seguida, pero con mucha menor presencia, del lentisco (Pistacia lentiscus), la cornicabra (Pistacia terebinthus), Pistacia sp. y el pino carrasco (Pinus halepensis) que mantienen porcentajes entre el 4-10\% en todos los yacimientos. El romero (Rosmarinus oficcinalis) y el aladierno (Phillyrea sp.) también muestran parecidos porcentajes en los tres yacimientos, aunque un poco más bajos (entre 1-3\%). El grupo formado por encina/coscoja (Quercus ilex-coccifera), álamos (Populus sp.), sauces (Salix sp.) y tarayes (Tamarix sp.) tienen porcentajes por debajo del $2 \%$, la mayoría menos de $1 \%$, salvo en alguno de los yacimientos con porcentajes en torno al 8\% (los tarayes en Zajara 7.91\%; los sauces $7.69 \%$ y la encina/coscoja $8.65 \%$ en Santa Bárbara). Las jaras (Cistus sp.), salados (Atriplex halimus) y las leguminosas sólo aparecen en Zajara y Campos, faltando en Santa Bárbara, presentan porcentajes por debajo del 1\% (salvo las leguminosas en Campos). Los torviscos (Daphne gnidium), belchos (Ephedra sp.) y monocotiledóneas, con escasa presencia, aparecen en Campos.

Por tanto, vemos que el número de taxones y su distribución porcentual entre los tres yacimientos es muy parecida aunque la mayor similitud se pro- duce entre Campos y Zajara (Fig. 2), que situados a unos $3 \mathrm{Km}$ de distancia en línea recta, presentan una misma imagen de la vegetación del entorno. Por contra, Santa Bárbara tiene ciertas singularidades en la representación de las distintas especies: el acebuche disminuye más de un tercio respecto a los dos yacimientos anteriores, y tiene porcentajes mayores para el pino carrasco, el aladierno, el lentisco, la Pistacia, la coscoja, el romero y el sauce. De todos estos los más significativos son la coscoja y el sauce que, con porcentajes por debajo del $1 \%$ en Campos y Zajara, presentan aquí valores en torno al $8 \%$. El mayor porcentaje de la coscoja junto a los romeros (que casi dobla su porcentaje) parece indicar la relativa mayor lejanía de la costa y la posible vecindad de formaciones vegetales termomesomediterráneas, como son los carrascales, lo cual podría indicar que el piso mesomediterráneo habría tenido su límite más bajo que actualmente, en las cercanías de Santa Bárbara (Fig. 1).

La ripisilva está representada con cuatro géneros: fresnos, álamos, sauces y tarayes, que presentan porcentajes por debajo del 1\% (indicados en la figura 2 por cuadrados), salvo el caso de los tarayes en Zajara y Campos y los sauces en Santa Bárbara. El conjunto de la vegetación de ribera presenta los siguientes porcentajes: Zajara 9.77\%, Campos $2.58 \%$ y Santa Bárbara 9.63\%. Estos porcentajes son relativamente bajos si pensamos que los yacimientos se encuentran ubicados junto a cursos de agua. Esta escasa presencia de especies higrófilas puede ser debida a la ocupación de los fondos de valle, área potencial de la ripisilva, por terrenos de 
cultivo, como ocurre en yacimientos calcolíticos y argáricos de la depresión de Baza-Huéscar (Rodríguez-Ariza, 1992b), aunque contrasta fuertemente con los resultados de Los Millares que tienen porcentajes en torno al $20 \%$ en el Fortín 1 y del $40 \%$ en el Poblado (Rodríguez-Ariza y Vernet, 1991; Rodríguez-Ariza, 1992a).

El predominio del acebuche en todos los yacimientos junto con el lentisco puede expresar una formación local termomediterránea de lentiscar, aunque la correspondencia con alguna de las series actualmente definidas es difícil, siendo la serie murciano-almeriense del Chamaeropo humilisRhamneto lycioidis sigmetum (AlcarazAriza y Peinado Lorca, 1987: 264; Rivas Martínez, 1988: 128) o la catalana-levantina del Oleo-Lentiscetum provinciale (Folch i Guillén, 1986: 122) las más próximas. También estaría presente, aunque probablemente en áreas reducidas o más o menos marginales, un matorral del Rosmarino Ericion junto con la presencia del pino carrasco.

La vegetación madura de las distintas asociaciones de lentiscares es un matorral esclerófilo donde predominan los lentiscos (Pistacia lentiscus) y acebuches (Olea europaea var. sylvestris), desarrollandose otras especies como palmitos (Chamaerops humilis), bayón (Osyris quadipartita), belchos (Ephedra fragilis), algarrobos (Ceratonia siliqua), etc. Son formaciones de matorral alto y espeso, con algún árbol ocasional, y cuya gran densidad las hace difícilmente penetrables. Estas entrarían dentro del término de maquis o maquia, actualmente muy debatido. Aquí podemos encontrar algunos pies de encina aislados y rodales de pino carrasco.

La intervención humana sobre estas asociaciones provoca la aparición de matorrales abiertos en los que las especies dominantes son las cistáceas, leguminosas como las fabáceas, brezos y algunas labiadas como romeros y tomillos.

Por tanto, podemos pensar que en el Bajo Almanzora en la Edad del Cobre existía un paisaje vegetal formado por un matorral más o menos denso de lentiscos y acebuches junto al que existían zonas, más o menos denudadas, donde estaría instalado un matorral abierto junto con algunos pinos carrascos. Los fondos de los valles estarían ocupados por campos de cultivo, habiendo reducido la vegetación natural a una estrecha banda junto al cauce.

Las formaciones de lentiscares se desarrollan bajo unos valores ombroclimáticos de semiárido superior al seco inferior (250-400 $\mathrm{mm}$ apr.) con unas temperaturas en torno a los $17^{\circ} \mathrm{C}$ de media anual, lo que corresponde al piso termomediterráneo, casi libre de heladas que sólo se pueden presentar entre los meses de diciembre-febrero.

Así, vemos que la zona que actualmente ocupa la asociación del azufaifo estaría ocupada durante la Edad del Cobre por la asociación del lentiscar (actualmente con un desarrollo potencial en el piedemonte de las Sierras de las Estancias, Filabres y Cabrera) y, por tanto, probablemente la vegetación mesomediterránea ocuparía el lugar de esta última. Esto supone que el límite entre los pisos termo- y mesomediterráneo estaría mucho más bajo que en la actualidad, en los entornos de los yacimientos situados a más altitud o al interior de la Depresión como son Santa Bárbara, Fuente Álamo y Gatas.

\section{III.2. La vegetación durante la Edad del Bronce}

\section{A. Fuente Álamo}

En el análisis antracológico de Fuente Álamo (Schoch y Schweingruber, 1982) se han identificado 12 taxones (Tab. 2), entre los que sobresale la Pistacia sp. (216 fragmentos de 505), seguida del Pinus sp. (96/505), el Tamarix sp. (86/505) y la Olea europaea (73/505). El resto de los taxones identificados, Atriplex cf. halimus, Tetraclinis articulata, Rhamnus sp., Erica cf. arborea, Viburnum tinus, Rosmarinus officinalis, Quercus ilex-coccifera y Ficus carica, están representados por menos de 10 fragmentos cada uno. Basándose en estos resultados los autores hablan de cuatro medios diferentes: maquia, vegetación hidrófila, ruderal y cultivos entre los que señalan a la higuera y el olivo, aunque sin indicar en que se basan para considerar la domesticación de ambas especies. Es de señalar la sola presencia de Tamarix sp. como representante de la ripisilva y la importancia de la maquia o los matorrales. Estos resultados se articulan bien con los del resto de yacimientos de la zona, aunque aquí la presencia de pino es más importante, quizás por la utilización de esta especie en la construcción de las casas argáricas (Rodríguez-Ariza, 1993), aunque sería deseable la realización de un estudio más minucioso donde se dilucidaran cuestiones como la determinación de las especies de pinos y Pistacia, así como un estudio cuantitativo que indicara la importancia de cada especie, a fin de determinar la posible formación vegetal dominante y por tanto la antropización del medio.

T. P., 57, n. ${ }^{\circ} 1,2000$ 


\begin{tabular}{|l|c|c|}
\hline \multicolumn{3}{|c|}{ FUENTE ALAMO } \\
\hline Taxones & $\mathrm{N}^{\circ}$ & $\%$ \\
\hline Atriplex sp. cf. halimus & 1 & 0.20 \\
\hline Erica sp. cf. arborea & 4 & 0.80 \\
\hline Ficus carica & 3 & 0.60 \\
\hline Olea europaea & 73 & 14.45 \\
\hline Pimus sp. & 96 & 19.00 \\
\hline Pistacia sp. & 216 & 42.77 \\
\hline Quercus coccifera/ilex & 1 & 0.20 \\
\hline Rhamnus sp. & 10 & 1.98 \\
\hline Rosmarinus officinalis & 2 & 0.40 \\
\hline Tamarix sp. & 86 & 17.02 \\
\hline Tetraclinis articulata & 9 & 1.78 \\
\hline Viburnum tinus & 4 & 0.80 \\
\hline $\mathrm{N}^{\circ}$ DE CARBONES & 505 & 100 \\
\hline $\mathrm{N}^{\circ}$ DE TAXONES & 12 & \\
\hline
\end{tabular}

Tab. 2. Frecuencias absolutas y relativas de los taxones determinados en el análisis antracológico de Fuente Álamo (Schoch y Schweingruber, 1982).

\section{B. Gatas}

Para realizar una primera valoración a nivel paleoecológico y de dinámica de la vegetación, nos hemos vistos obligados a agrupar los resultados de las diferentes tablas (Gale, 1999: apéndice 2) por períodos cronológicos, y en aquellos casos en que había suficiente cantidad de carbón (Bronce Argárico y Bronce Tardío) calcular las frecuencias relativas de los diferentes taxones para poder comparar y evaluar los posibles cambios de la vegetación a través de la secuencia cronológica prehistórica (Tab. 3). Ahora bien, esta aproximación no está exenta de dificultades, ya que al desconocer los contextos arqueológicos en. los que fueron recuperadas las distintas muestras antracológicas, la interpretación se puede ver afectada por múltiples factores tanto deposicionales como postdeposicionales (Rodríguez-Ariza, 1993).

El número de taxones determinados por Rowena Gale en los niveles prehistóricos de Gatas se eleva a 23, de ellos 8 aparecen sólo en un período cultural: Cistus, Ericaceae, Myrtus, Nerium, Pomoideae/Prunus, Teucrium y Salicaceae en el Bronce Argárico y Spartium junceum en el Bronce Tardío, estando la mayoría representados solamente por un fragmento de carbón, lo cual siempre habrá de ser tenido en cuenta a la hora de realizar una valoración

\begin{tabular}{|c|c|c|c|c|c|c|c|}
\hline \multicolumn{8}{|c|}{ GATAS } \\
\hline \multirow{3}{*}{ TAXONES/FASES } & \multicolumn{4}{|c|}{ Argar } & \multicolumn{2}{|c|}{ Post-Argar } & \multirow{3}{*}{$\begin{array}{l}\text { Bronce } \\
\text { Final }\end{array}$} \\
\hline & \multirow[t]{2}{*}{ Poblado } & \multirow[t]{2}{*}{ Sep. } & \multicolumn{2}{|c|}{ Total } & \multirow[t]{2}{*}{$\mathrm{N}^{\circ}$} & \multirow[t]{2}{*}{$\%$} & \\
\hline & & & $\mathrm{N}^{\circ}$ & $\%$ & & & \\
\hline Cistus & 2 & 3 & 5 & 0.50 & - & - & - \\
\hline Ericaceae & 5 & - & 5 & 0.50 & - & - & - \\
\hline Ficus & 22 & 8 & 30 & 2.88 & 3 & 0.70 & 7 \\
\hline Leguminosae & 11 & 1 & 12 & 1.15 & 2 & 0.46 & 6 \\
\hline Myrtus & - & 1 & 1 & 0.10 & - & - & - \\
\hline Nerium & - & 1 & 1 & 0.10 & - & - & - \\
\hline Olea & 396 & 33 & 429 & 41.28 & 260 & 60.70 & 25 \\
\hline Phillyrea/Rhamnus & 12 & - & 12 & 1.15 & 2 & 0.46 & - \\
\hline Pinus & 208 & 4 & 212 & 20.40 & 5 & 1.16 & 11 \\
\hline Pistacea & 34 & 8 & 42 & 4.04 & 91 & 21.20 & 1 \\
\hline Pistacea/Rhus & 10 & - & 10 & 0.96 & 19 & 4.43 & 2 \\
\hline Pomoidae/Prunus & 1 & - & 1 & 0.10 & - & $=$ & - \\
\hline Populus/Salix & 12 & 3 & 15 & 1.44 & 3 & 0.70 & - \\
\hline Prunus & 3 & 3 & 6 & 0.59 & 3 & 0.70 & - \\
\hline Quercus & 154 & 7 & 161 & 15.49 & 7 & 1.60 & 9 \\
\hline Quercus suber & 40 & - & 40 & 3.84 & 5 & 1.16 & - \\
\hline Rhamnus & 6 & 4 & 10 & 0.96 & 1 & 0.23 & - \\
\hline Rosmarinus & 3 & 5 & 8 & 0.76 & 3 & 0.70 & - \\
\hline Rhus & 2 & - & 2 & 0.20 & 3 & 0.70 & - \\
\hline Salicaceae & 7 & - & 7 & 0.67 & - & - & - \\
\hline Spartium junceum & - & - & - & - & 7 & 1.60 & - \\
\hline Tamarix & 8 & 19 & 27 & 2.59 & 15 & 3.50 & 1 \\
\hline Teucrium & 3 & - & 3 & 0.30 & - & - & - \\
\hline $\mathrm{N}^{\circ} \mathrm{CARBONES}$ & 939 & 99 & 1038 & 100 & 428 & 100 & 62 \\
\hline TOTAL TAXONES . & 20 & 14 & 22 & & 16 & & 8 \\
\hline
\end{tabular}

Tab. 3. Frecuencias absolutas y relativas de los taxones determinados en el análisis antracológico de Gatas (elaboración propia a partir de Gale, 1999).

paleoecológica o paleoetnológica. Estos ocho taxones entran dentro de la flora típica de la zona.

Por tanto, si quitamos estos 8 taxones, raros o excepcionales, la lista se queda reducida a 15 todos ellos muy abundantes en el resto de yacimientos de la zona, salvo los casos de Prunus sp., Rhus y Quercus suber. Los dos primeros en muy escaso número y el último con una presencia significativa en el Bronce Argárico y Tardío, habiendo aparecido no sólo carbón, sino también corcho.

El catálogo florístico de Gatas (Tab. 3) está compuesto por árboles: pinos (sin determinar la especie, aunque se señala en el texto que posiblemente sea el pino carrasco), encinas o coscojas, alcornoques e higueras (estas posiblemente cultivadas, aunque a nivel anatómico es imposible su determinación). Los arbustos están representados por el acebuche, la coscoja, el lentisco y/o la cornicabra y rosáceas del género Prunus; y por especies del matorral fruticoso: jaras, brezos, leguminosas, aladiernos y/o espinos y romeros. La vegetación de los cursos de agua sólo está compuesta por álamos, adelfas, sauces y tarayes, con una presencia escasa.

En la figura 3 hemos comparado las frecuencias relativas de los 6 taxones con mayores porcentajes (Olea, Pinus, Pistacia, Quercus, Q. suber y Tamarix) en el Argar y Postargar. En primer lugar hay que destacar la predominancia de la Olea en los dos 


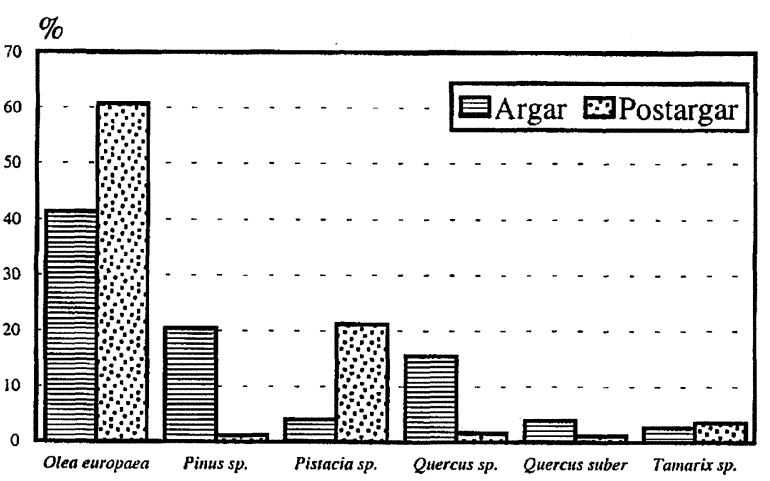

Fig. 3. Comparación de los porcentajes de los taxones mayores determinados en el análisis antracológico de Gatas.

períodos, con porcentajes que duplican o triplican al segundo representado. La distinción anatómica entre el olivo y el acebuche (Olea europea var. sylvestris) es muy difícil de realizar, aunque algunos intentos se han hecho utilizando diversos parámetros anatómicos que han sido tratados por análisis multivariantes (Terral y Arnold-Simard, 1996; Terral, 1997). En segundo lugar hay que señalar que en el Argar junto a la Olea los taxones más representados con porcentajes muy significativos son los pinos $(20.40 \%$ ) y los Quercus (posiblemente encina o coscoja) con el $15.49 \%$, mientras que en el Postargar estos disminuyen al $1 \%$ y dejan su lugar a las Pistacia con el $2.20 \%$ de representación. Este hecho nos plantea varias hipótesis: por un lado, la fuerte representación de los pinos en el Argar puede deberse a su utilización como elemento de construcción como ocurre en otros yacimientos argáricos (Rodríguez-Ariza, 1992a) y su casi desaparición en el Bronce Tardío al cambio de los sistemas constructivos, aunque, como ha sido señalado con anterioridad, el desconocimiento de donde proceden las muestras antracológicas nos impide poder contrastar esta hecho. Por otro lado, estos dos grupos de taxones podrían estar representando a distintas asociaciones vegetales: así, los pinos se asocian principalmente a la encina/coscoja y a todas las especies representativas de un desarrollo más o menos significativo del matorral fruticoso (jaras, brezos, espinos, romeros y leguminosas). Parece ser que en este momento existe una vegetación tipo maquia con un fuerte predominio de la coscoja y la carrasca y un matorral del Rosmarino Ericion junto con la presencia del pino carrasco, pudiendo incluso aventurar la hipótesis que los carrascales basófilos termomesomediterráneos, que actualmente sólo se encuentran en las partes altas de Sierra Cabrera re- presentados por la serie Smilaci mauritanicaeQuerceto rotundifoliae (Fig.1), descendieran hasta las inmediaciones del yacimiento.

La asociación del acebuche con el lentisco en el Postargar viene acompañada por una desaparición de la mayoría de las especies del matorral o una disminución de sus frecuencias relativas, expresando una formación vegetal de la series de los lentiscares.

Por tanto, el cambio en la composición vegetal entre el Argar y el Postargar (de un carrascal a un lentiscar) podría indicar un cambio en las condiciones bioclimáticas principalmente en el ombroclima, tendente a una mayor sequedad, y/o un cambio de la acción antrópica sobre el medio.

\section{LA DINÁMICA DE LA VEGETACIÓN DURANTE LA PREHISTORIA RECIENTE}

La tabla 4 muestra los taxones determinados en cada uno de los yacimientos y períodos culturales que cuentan con análisis antracológicos en la zona.

El primer comentario que hay que realizar es sobre la gran cantidad de taxones determinados (37), aunque si eliminamos los taxones dobles (Phillyrea/Rhamnus, Pistacea/Rhus, Pomoidea/Prunus y Populus/Salix) que están representados en la lista por sus dos componentes el número se queda reducido a 33. Los Pinus sp., que se determinan en Gatas y Fuente Álamo, habría que unirlos, con toda probabilidad, al Pinus halepensis que aparece en los yacimientos calcolíticos, al igual que ocurre con la Pistacia sp. que se podría adjudicar a Pistacia lentiscus o Pistacia terebinthus y los Quercus sp. que creemos que podrían corresponderse con el Quercus ilex-coccifera, por lo cual tendríamos 30. Además, si eliminamos los 11 taxones simples que aparecen en un sólo yacimiento y período cultural (Daphne gnidium, Ephedra, Fraxinus, Myrtus, Nerium, Pinus pinaster, Spartium junceum, Tetraclinis articulata, Teucrium, Viburnum tinus y Monocotiledoneae) la lista se nos vuelve a reducir a 19 taxones, que serían los taxones que nos pueden definir con mayor exactitud las distintas asociaciones vegetales desarrolladas a lo largo del tiempo en la Depresión de Vera.

De este grupo, 4 taxones están presentes en todos los yacimientos y períodos culturales, constituyéndose en los indicadores vegetales de la zona. Estos son: Olea europaea, Pinus halepensis, Pistacea lentiscus y Quercus ilex-coccifera. Junto a estos el 


\begin{tabular}{|c|c|c|c|c|c|c|c|}
\hline \multicolumn{8}{|c|}{ ANÁLISIS ANTRACOLÓGICOS DE LA DEPRESIÓN DE VERA } \\
\hline Secuencia Cultural & & COBRE & & $\begin{array}{r}\text { BR } \\
\text { PLEN }\end{array}$ & $\begin{array}{l}\text { NCE } \\
\text { (Argar) }\end{array}$ & $\begin{array}{l}\text { BRONCE } \\
\text { TARDÍO }\end{array}$ & $\begin{array}{l}\text { BRONCE } \\
\text { FINAL }\end{array}$ \\
\hline Yacimientos & Zajara & Campos & \begin{tabular}{|c|} 
Santa \\
Bárbara
\end{tabular} & Gatas & $\begin{array}{l}\text { Fuente } \\
\text { Álamo }\end{array}$ & Gatas & Gatas \\
\hline Atriplex halimus (salados) & * & * & & & * & & \\
\hline Cistus sp. (jaras) & * & * & & * & & & \\
\hline Daphne gnidium (torvisco) & & * & & & & & \\
\hline Ephedra sp. (belchos) & & * & & & & & \\
\hline Ericaceae (brezos) & & & & * & * & & \\
\hline Ficus carica (higuera) & & & & * & * & * & * \\
\hline Fraximus sp. (fresnos) & * & & & & & & \\
\hline Leguminosae (leguminosas arbustivas) & * & * & & * & & $*$ & * \\
\hline Monocotiledoneae (Monocotiledóneas) & & * & & & & & \\
\hline Myrtus (mirto) & & & & * & & & \\
\hline Nerium (adelfas) & & & & * & & & \\
\hline Olea europaea (acebuche) & * & * & $*$ & $*$ & $*$ & $*$ & $*$ \\
\hline Phillyrea sp. (aladiernos) & * & * & $*$ & & & & \\
\hline Phillyrea/Rhamnus & & * & & * & & $*$ & \\
\hline Pimus sp. (pinos) & & * & $*$ & * & $*$ & $*$ & $*$ \\
\hline Pinus halepensis (pino carrasco) & * & * & * & & & & \\
\hline Pinus pinaster (pino maritimo) & & & * & & & & \\
\hline Pistacea & $*$ & $*$ & * & * & $*$ & $*$ & $*$ \\
\hline Pistacia lentiscus (lentisco) & * & $*$ & $*$ & & & & \\
\hline Pistacia terebinthus (cornicabra) & $*$ & * & $*$ & & & & \\
\hline Pistacea/Rhus & & & & $*$ & & $*$ & $*$ \\
\hline Pomoidae/Prunus & & & & * & & & \\
\hline Populus sp. (álamos) & * & * & $*$ & & & & \\
\hline Populus/Salix & & & & $*$ & & $*$ & \\
\hline Prumus sp. (prunoideas) & & & & * & & * & \\
\hline Quercus sp. & & & & * & & * & $*$ \\
\hline Quercus ilex-coccifera (encina-coscoja) & * & $*$ & $*$ & & * & & \\
\hline Quercus suber (alcornoque) & & & & * Corcho & & *Corcho & \\
\hline Rhamnus sp. (espinos) & & & & * & * & * & \\
\hline Rosmarinus officinalis (romero) & $*$ & * & $*$ & $*$ & * & * & \\
\hline Rhus (zumaque) & & & & $*$ & & $*$ & \\
\hline Salix sp. (sauces) & $*$ & $*$ & * & $*$ & & & \\
\hline Spartium junceum (gayomba) & & & & & & * & \\
\hline Tamarix sp. (tarayes) & $*$ & $*$ & * & $*$ & * & $*$ & $*$ \\
\hline Tetraclinis articulata (tuya) & & & & & $*$ & & \\
\hline Teucrium (romerillo) & & & & $*$ & & & \\
\hline Viburnum timus (durillo) & & & & & $*$ & & \\
\hline TOTAL TAXONES & 15 & 19 & 13 & 22 & 12 & 16 & 8 \\
\hline
\end{tabular}

Tab. 4. Distribución en los distintos yacimientos y períodos culturales de los taxones determinados en los análisis antracológicos de la Depresión de Vera. 
Rosmarinus officinalis y Tamarix sp. sólo faltan en un período de Gatas y las Leguminosae en Santa Bárbara y Fuente Álamo. Este grupo de taxones nos define una vegetación termófila con predominancia de los arbustos de gran talla como los acebuches, el lentisco y la coscoja, junto a un matorral representado por romeros y leguminosas arbustivas y la presencia de dos árboles, aunque también adoptan porte arbustivo, como son el pino carrasco y los tarayes. Por tanto, en principio podríamos pensar que la vegetación predominante es la de tipo maquia o garriga, descrita para las zonas costeras de diversas regiones mediterráneas, lo cual ya contrasta con la vegetación actual (prácticamente inexistente) e incluso con la vegetación potencial de la zona constituida por cornicales y espinales (Fig. 1).

$\mathrm{Si}$ analizamos los datos a nivel cronológico, además de lo comentado para cada yacimiento, hay que señalar la casi desaparición de la ripisilva que constituída en la Edad del Cobre por fresnos, álamos, sauces y tarayes, sólo está representada por estos últimos en la Edad del Bronce junto a una escasa presencia de álamos o sauces en el Argar y Postargar de Gatas. Este fenómeno de desaparición de la ripisilva podría indicar un cambio de los parámetros bioclimáticos tendentes a una menor humedad, lo cual provocaría una disminución del caudal de los cursos de agua y, por tanto, de la vegetación asociada.

También hay que destacar la aparición de la higuera en Fuente Álamo y Gatas a partir del Bronce Pleno, lo cual abogaría por el cultivo de esta especie a partir de esta época, aunque hay que tener en cuenta que esta especie es característica de la vegetación mediterránea y la diferenciación anatómica entre la especie cultivada y la silvestre es imposible de realizar. La aparición de una cantidad significativa de carbón en Gatas y carbón y semillas en Fuente Álamo (Stika, 1988) nos lleva a poder plantear que esta zona fuera un foco de domesticación o al menos de introducción del cultivo de esta especie en la Península Ibérica, aunque en la zona estuviera de forma natural con anterioridad.

Resumiendo, podríamos afirmar que el predominio del acebuche en todos los yacimientos junto con el lentisco puede expresar una formación local termomediterránea de lentiscar, aunque en algunos yacimientos como Santa Bárbara y en el Bronce Pleno de Gatas está representada una vegetación más mesófila, indicando posiblemente que el piso de vegetación mesomediterráneo bajaría hasta las inmediaciones de estos yacimientos. A partir del Bronce Tardío parece asistirse a un cierto cambio en la vegetación, tendente a una mayor aridez, aunque las temperaturas parecen que no sufren variaciones importantes.

\section{LA DEPRESIÓN DE VERA DENTRO DE LA DINÁMICA PALEOECOLÓGICA DEL MEDITERRÁNEO OCCIDENTAL}

En la zona de estudio o en comarcas próximas se vienen desarrollando en la última década una serie de actuaciones arqueológicas, que han dado como resultado la existencia de un corpus de datos antracológicos y polínicos que brevemente pasamos a describir:

En la misma Depresión de Vera contamos con los datos preliminares del antracoanálisis del yacimiento calcolítico de Las Pilas (Mojácar, Almería) (3) en el que se han identificado 16 taxones, entre los que destacan: Olea europaea, Pistacia lentiscus, Pistacia sp. y Quercus ilex-coccifera. También aparecen pero en porcentajes muy pequeños: Pinus halepensis, Pinus sp., Cistus sp., Leguminosas, Phillyrea sp., Pistacia terebinthus, Rhamnus sp. y Rosmarinus officinalis y entre la ripisilva: Fraxinus sp., Populus sp. y Tamarix sp. Este catálogo florístico coincide plenamente con el de los yacimientos aquí estudiados, dando una misma imagen de la vegetación en la Edad del Cobre en todo el área de la Depresión de Vera y el BajoAlmanzora.

También contamos con el análisis antracológico del poblado argárico del Rincón de Almendricos (Grau, 1990) situado en la comarca de Lorca, actualmente, en el piso de vegetación Termomediterráneo con un ombroclima semiárido inferior, muestra que durante el período subboreal en el que se desarrolló la cultura del Argar, habría en los alrededores del yacimiento una vegetación termófila caracterizada por la presencia de encina, pino carrasco, acebuche y brezo. La autora señala que estas especies son indicadoras de un cierto estado de degradación de los carrascales continentales (Bupleuro rigidi-Quercetum rotundifoliae sigmetum) como podría ser el Rhamno lycioidis-Querceto cocciferae sigmetum. Esta asociación, señalada por nosotros para definir la vegetación calcolítica de la

(3) El material antracológico procede de las excavaciones de urgencia realizadas en el yacimiento por un equipo formado por miembros del Dpto. de Prehistoria y Arqueología de la Universidad de Granada y del Dpto. de Prehistoria, Antropología e Historia Antigua de la Universidad de la Laguna. 
Depresión de Baza-Huéscar, evolucionará en época argárica hacia un matorral abierto rico en pino carrasco, aunque aquí no estén presentes taxones termófilos como el acebuche, por ser una zona más continental (Rodríguez-Ariza, 1992a,b).

El estudio antracológico del Cerro de las Viñas (Grau, 1990), poblado situado dentro del piso de vegetación mesomediterráneo inferior con un ombroclima semiárido superior, en el término de Lorca (Murcia), muestra una vegetación similar a la de la altiplanicie de Baza-Huéscar. Aquí domina el Pinus halepensis, seguido del Quercus ilex-coccifera y las Leguminosas. También se han identificado Erica multiflora, Juniperus sp., Rhamnus lycioides, Pistacia lentiscus y Tetraclinis articulata.

En estudios polínicos realizados en la vertiente mediterránea de la Península Ibérica por un grupo de la Universidad Autónoma de Barcelona, que han publicado las secuencias holocenas de Roquetas de Mar, Antas y San Rafael (Pantaleón-Cano et alii, 1999), sitúan el óptimo holoceno entre los 7000 y los $4500 \mathrm{bp}$, en el que las formaciones arbustivas se desarrollarían ampliamente en las zonas bajas mientras que formaciones forestales más mesófilas se extenderían en las áreas montañosas interiores. A partir del 4500 bp se establece una vegetación más estépica como consecuencia de unos condiciones ambientales más áridas.

Así en el diagrama polínico de Roquetas de Mar se refleja un gradual y constante retroceso de los taxones arbóreos, especialmente de Quercus caducifolio, Quercus perennifolio y Olea, dominantes en la base y cuyo descenso es compensado sólo en parte por la notable subida de Pinus.

En un artículo de B. Mariscal (1991) sobre el Holoceno en Las Pilas de Mojácar, se realiza un análisis polínico de sedimentos de la zona, sin indicar la situación, estratigrafía y cronología de los sondeos, por lo cual su validez es nula.

Los análisis polínicos realizados dentro del Proyecto Aguas (Stevenson, 1998) en Cortijo del Campo, Balsa de Marchalico y Balsa de Alquirrico de los Peñones muestran una gran pobreza de polen arbóreo, sólo representado por Olea y Pinus.

En el análisis polínico de Almizaraque (López, 1988) la autora observa dos períodos:

-el inferior en el que domina el Pinus halepensis, acompañado por taxones mediterráneos como Buxus sempervirens o Vitis vinifera, quedando atestiguados cultivos agrícolas, Cerealia, junto a Fabáceas, Plantago y Poligonáceas.

-El superior, en el que los pinos disminuyen, y los espacios abiertos se ven ocupados por Asteráceas ligulifloras y Quenopodiáceas. Según la autora esta fase corresponde con el abandono del yacimiento, con una vegetación próxima a la de la actualidad del tipo xerófilo propia de un clima extremadamente seco.

A partir de los estudios antracológicos aquí presentados podemos decir que la vegetación de la Depresión deVera durante el III milenio a.n.e. estaba dominada por comunidades arbustivas instaladas en el centro o zonas bajas, mientras que las formaciones arbóreas se situarían en los piedemontes de las sierras cercanas. Durante el II milenio parece que existe un progresivo aumento de la aridez, que hace que las formaciones arbustivas vayan siendo sustituidas por matorrales abiertos y las formaciones arbóreas se queden relegadas en las sierras.

Estos resultados nos hablan de una correspondencia de la vegetación a nivel regional, donde se aprecia un cambio significativo de la vegetación entre la Edad del Cobre y Edad del Bronce, que en el Cerro de la Virgen se produce de modo paulatino (Rodríguez-Ariza, 1992b). El origen de este creemos que está en la influencias de origen antrópico por el desfase cronológico observado con datos paleoambientales de otras regiones, así en el sur de Francia (Vernet y Thiébault, 1987) y Levante peninsular (Vernet et alii, 1983, 1987; Badal, 1990) éste proceso se inicia en el Neolítico Medio. Mientras que en la Depresión de Ronda la abertura de la vegetación no parece producirse hasta comienzos del I milenio a. C. (Rodríguez-Ariza et alii, 1992c). Evidentemente las influencias de cambio climático, como señalan los estudios polínicos anteriormente citados son importantes, pero el comienzo y ritmo de las transformaciones del paisaje en una comarca determinada vienen condicionadas por la mayor o menor incidencia de la acción antrópica sobre el medio.

\section{BIBLIOGRAFÍA}

Alcaraz Ariza, F. y Peinado Lorca, M. (1987): "El Sudeste ibérico semiárido". En M. Peinado y S. Rivas (eds.): La vegetación de España. Universidad de Alcalá de Henares. Alcalá de Henares: 259-281.

Badal García, E. (1990): Aportaciones de la Antracología al estudio del paisaje vegetal y su evolución en el cuaternario reciente, en la costa mediterránea del Pais valenciano y Andalucía (18.000-3.000 B.P.). Tesis doctoral microfilmada. Universidad de Valencia. Valencia.

T. P., 57, n. ${ }^{\circ} 1,2000$ 
Castro, P.V; Chapman, R.W; Gili, S.; Lull, V; Micó, R; Rinuete, C.; Risch, R. y Sanahuja, M.E. (eds.) (1998): Aguas Project. Palaeoclimatic reconstruction and dynamics of human settlement and land-use in the area of middle Aguas (Almería), in the south-east of the Iberian Peninsula. European Commission Environment and Climate Programme. Luxembourg.

FERnández MarTínez, V.M. (1989): Teoría y método de la Arqueología. Editorial Síntesis. Madrid.

FolCh GuILLÉn, R. (1986): La vegetacio dels països catalans. Ed. Ketres. Barcelona.

Gale, R. (1999): "Gatas: Análisis antracológico". En P.V. Castro, R.W. Chapman, S. Gili, V. Lull, R. Micó, C. Rihuete, R. Risch y M.E. Sanahuja (eds.): Proyecto Gatas 2. La dinámica arqueológica de la ocupación prehistórica. Arqueología Monografías. Junta de Andalucía. Sevilla: 292-307.

Grau Almero, E. (1990): El uso de la madera en yacimientos valencianos de la Edad del Bronce a la época visigoda. Datos etnobotánicos y reconstrucción ecológica según la antracología. Tesis doctoral microfilmada. Universidad de Valencia. Valencia.

Leroi-Gourhan, A. (1992): "Bois et pollens: étude complémentaire". Bulletin de la Société Botanique de France, 139. Actualites botaniques (2/3/4): 273-280.

LÓPEZ, P. (1988): "Estudio polínico de seis yacimientos del Sureste español". Trabajos de Prehistoria, 45: 335345.

MARISCAL, B. (1991): "Características ambientales durante el Holoceno en la Pilas de Mójacar, Almería. Análisis polínico en la Cuenca del ríoAguas". Boletín Geológico y Minero, 102-3: 394-399.

Pantaleón-Cano, J.; Yll, R. y Roure, J.M. (1999): “Evolución del paisaje vegetal en el sudeste de la Península Ibérica durante el Holoceno a partir del análisis polínico". En II Congrés del Neolitic a la Península Ibérica. Saguntum-Plav. Extra-2: 17-23.

Peinado Lorca, M. y Rivas Martínez, S. (eds.)(1987): La vegetación de España. Universidad deAlcalá de Henares. Alcalá de Henares.

Rivas MartíneZ, S. (1988): Memoria del mapa de Series de vegetación de España 1:400.000. ICONA, Madrid.

RodrígueZ-ARIZA, M. ${ }^{\mathrm{a} O}$. (1992a): Las relaciones hombrevegetación en el Sureste de la Península Ibérica durante las Edades del Cobre y Bronce a partir del análisis antracológico de siete yacimientos arqueológicos. Tesis doctoral microfilmada. Universidad de Granada.

- (1992b): "Human-plant relationships during the Copper and Bronze Ages in the Baza and Guadix Basins (Granada, Spain)". Bulletin de la Société Botanique de France, 139. Actualites botaniques (2/3/4): 451-464.

- (1993): "Los procesos de formación y transformación del registro arqueológico en los estudios antracológicos". Arqueología Espacial, 16-17: 371-390.

Rodríguez-Ariza, Ma .O.; Aguayo, P. y Moreno, F. (1992c): "The environment in the Ronda Basin (Malága, Spain) based on an anthracological study of Old Ronda". Societé Botanique de France, 139. Actualites botaniques (2/3/4): 715-725.

RodríGueZ-ArizA, Mão. y Vernet, J.L. (1991): "Etude paleoecologique du Gisement Chalcolithique de Los Millares (Santa Fé de Mondujar, Almería). EtudeAnthracologique". En W.H. Waldren, J.A. Ensenyat y R.C. Kennard (eds.): Recent Developments in Western Mediterranean Prehistory: Archaeological Techniques, Technology and Theory. BAR International Series, 573. Oxford: 1-16.

Schoch, W. y Schweingruber, F. H. (1982): "Holzkohlenalytische ergebnisse aus der bronzezeitlichen siedlung Fuente Alamo. Prov. Almeria. Spanien". Archäologisches Korrespondenzblatt, 12: 451-455.

STEVEnson, A.C. (1998): "Pollen analysis in southeast Spain”. En P.V. Castro, R.W. Chapman, S. Gili, V. Lull, R. Micó, C. Rihuete, R. Risch y M.E. Sanahuja (eds.): Aguas Project. Palaeoclimatic reconstruction and dynamics of human settlement and land-use in the area of middle Aguas (Almería), in the south-east of the Iberian Peninsula. European Commission Environment and Climate Programme. Luxembourg: 62-65.

STIKA, H.P. (1988): "Botanische Untersuchungen in der bronzezeitlichen Höhensiedlung Fuente Alamo". Madrider Mitteilungen, 29: 21-76.

Terral, J.F. (1997): "Débuts de la domestication de l'olivier (Olea europaea L.) en Méditerranée nord-occidentale, mise en évidence par l'analyse morphométrique appliquée à du matériel anthracologique". Académie des Sciences/Elsevier, 324, IIa: 417-425.

Terral, J.F. y ARnold-Simard, G. (1996): "Beginnings of Olive Cultivation in Eastern Spain in relation to Holocene bioclimatic changes". Quaternary Research, 46: 176-185.

Vernet, J.L.; Badal García, E. y Grau, E. (1983): “La végétation néolithique du sud-est de l'Espagne (Valencia, Alicante) d'après l'analyse anthracologique". Académie des Sciences/Elsevier, 296, III: 669-672.

- (1987): "L'environnement végétal de l'homme au Néolithique dans le sud-est de L'Espagne (Valence, Alicante), première synthese d'après l'analyse anthracologique". Actes du Colloque "Premières communautés paysannes en Mediterranée occidentale" (Montpellier, 1983). CNRS. Montpellier: 131-134.

VERnET, J.L. y ThiÉBAuLt, S. (1987): “An approach to northwestern Mediterranean recent prehistoric vegetation and ecologic implications". Journal of Biogeography, 14: 117-127.

Vernet, J.L.; Thiébault, S. y Heinz, C. (1987): “Nouvelles données sur la végétation préhistorique postglaciar méditerranéenne d'après l'analyse anthracologique". Actes $d$ u colloque "Premières communautés paysannes en Mediterranée occidentale” (Montpellier, 1983). CNRS. Montpellier: 87-94.

T. P., 57, n. $^{\circ} 1,2000$ 